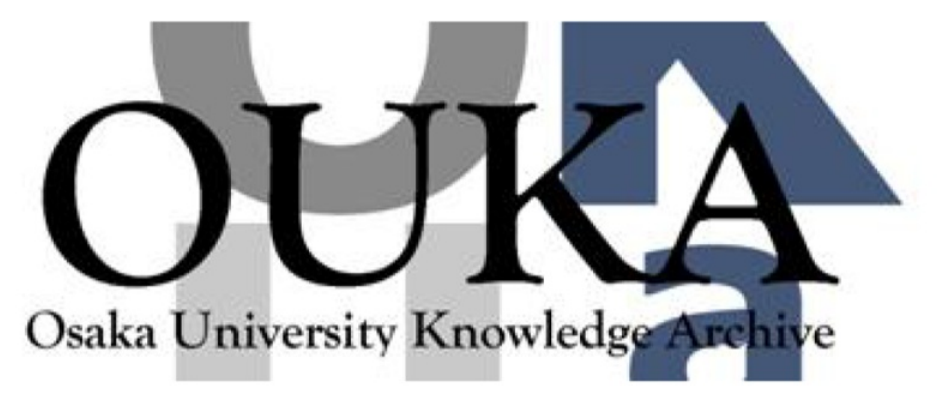

\begin{tabular}{|c|l|}
\hline Title & $\begin{array}{l}\text { Large-scale molecular dynamics simulations of } \\
\text { cluster impact and erosion processes on a } \\
\text { diamond surface }\end{array}$ \\
\hline Author(s) & Yamaguchi, Yasutaka; Gspann, Jürgen \\
\hline Citation & Physical Review B. 66(15) p. 155408 \\
\hline Issue Date & $2002-10-04$ \\
\hline oaire:version & VoR \\
\hline URL & https://hdl. handle. net/11094/82377 \\
\hline rights & Copyright 2002 by the American Physical Society \\
\hline Note & \\
\hline
\end{tabular}

Osaka University Knowledge Archive : OUKA

https://ir. library. osaka-u. ac. jp/

0saka University 


\title{
Large-scale molecular dynamics simulations of cluster impact and erosion processes on a diamond surface
}

\author{
Yasutaka Yamaguchi* \\ Department of Mechanical Engineering, The University of Tokyo, 7-3-1 Hongo, Bunkyo, Tokyo 113-8656, Japan \\ Jürgen Gspann ${ }^{\dagger}$ \\ Institut für Mikrostrukturtechnik, Universität Karlsruhe, Postfach 3640, D-76021 Karlsruhe, Germany
}

(Received 6 March 2002; revised manuscript received 21 June 2002; published 4 October 2002)

\begin{abstract}
Single $\mathrm{Ar}_{n}$ or $\left(\mathrm{CO}_{2}\right)_{n}(n \simeq 960)$ cluster impacts on a diamond (111) surface are studied by large-scale molecular dynamics simulations in order to investigate highly energetic cluster-surface interactions. For a cluster impact energy $E_{a}$ of $100 \mathrm{keV}$, a hemispherical crater and multilayered shockwaves are observed. Rebounding hot fluidized carbon material is seen to replenish the transient crater very quickly, with a central peak appearing as a long time phenomenon in the case of a $\mathrm{CO}_{2}$ cluster impact. Transient craters develop also for lower impact energies of $30 \leqslant E_{a} \leqslant 75 \mathrm{keV}$ while only an elastic deformation is observed for $E_{a}$ $=10 \mathrm{keV}$. The volume of the transient crater is approximately proportional to $E_{a}$ while the volume of the plastically deformed region and the kinetic energy transfer via the shockwave are linear functions of $E_{a}$ minus a threshold energy of about $10 \mathrm{keV}$. At an impact energy of $100 \mathrm{keV}$, the number of carbon atoms emitted from the target is much larger for a $\mathrm{CO}_{2}$ cluster impact than for an Ar cluster impact with a factor of about 3.35. The reactive enhancement of the surface erosion in the $\mathrm{CO}_{2}$ case is also proven by a strong $\mathrm{CO}$ signal in the spectrum of the emitted fragments. On the other hand, the surface of the relaxed crater is more densely packed and smoother in the case of the Ar cluster impact.
\end{abstract}

DOI: $10.1103 /$ PhysRevB.66.155408

PACS number(s): 79.20.Rf, 31.15.Qg

\section{INTRODUCTION}

Atomic and molecular cluster beams are considered useful tools for microscale and nanoscale surface modification including deposition, ion implantation, and physical as well as chemical erosion. Thin film growth via ionized cluster beam (ICB) deposition has been aimed at in particular. ${ }^{1,2}$ It is actually possible to obtain intense beams of neutral clusters from pure vapor expansions of cesium, zinc, silver, and, for periods of minutes, gallium by using feed vapor pressures in the range of bar, ${ }^{3}$ however, in order to fully achieve the original ICB deposition concept, a more effective broad beam ionization and the acceleration of such high-intensity metal beams are still required.

On the other hand, gas cluster beams can easily be generated from high-density source gas conditions, ${ }^{4}$ as has been long and deeply studied for nuclear fusion purposes. ${ }^{5}$ The authors' group applied highly accelerated ionized cluster beams as a tool for microscale and nanoscale surface structuring. ${ }^{6-8}$ In this technique, clusters consisting of about $1000 \mathrm{CO}_{2}$ molecules or Ar atoms, respectively, are accelerated to $100 \mathrm{keV}$. The impact-induced high energy density creates a plasma of cluster and surface material which may be highly reactive, in the case of the $\mathrm{CO}_{2}$ cluster impact. Therefore, the technique is called RACE (reactive accelerated cluster erosion). According to secondary electron microscope observations, very smooth eroded surfaces are obtained

with diamond, silicon, glass, and Teflon film. Among others, especially with artificial chemical vapor deposition diamond, the erosion results in an appreciable surface smoothing, or planarization, ${ }^{6,7}$ which seems to be attributable to a kind of fluidization of the surface material. Experiments using non-reactive argon clusters yield erosion rates about 4 times lower than those observed with $\mathrm{CO}_{2}$ clusters. In addition, atomic force microscope investigations revealed hillocks of about 1-2 nm height as a result of single $\mathrm{CO}_{2}$ cluster impacts on polished silicon. ${ }^{6}$ On natural single crystal diamond, on the other hand, erosion with non-reactive argon clusters results in an appreciably smoother surface than $\mathrm{CO}_{2}$ cluster erosion, even smoother than the original (111) surface of natural diamond. Hence accelerated cluster erosion techniques are especially suitable tools for direct micromachining.

A number of molecular dynamics studies on clustersurface interactions have been reported in the last decade: on the impinging cluster itself, ${ }^{9}$ on cluster deposition, ${ }^{10,11}$ implantation, ${ }^{12}$ or sputtering (surface erosion). ${ }^{13,14}$ Among these contributions, some studies considering higher acceleration energies focused on the emission, ${ }^{14}$ crater size, ${ }^{15}$ or implantation depth. ${ }^{16}$

In this paper, molecular dynamics simulations of cluster impacts on a diamond surface are performed with high acceleration energy $E_{a}$ up to $100 \mathrm{keV} / \mathrm{cluster}$ in order to investigate the surface erosion process, and the differences between inert argon and reactive $\mathrm{CO}_{2}$ cluster impacts are discussed in terms of the surface structure and the erosion effect in detail. In addition, the dependence of the transient crater size, the energy transfer via shockwave and the size distribution as well as the emission angle of the emitted fragments on the cluster acceleration energy are examined. The empirical potential function proposed by Brenner ${ }^{17}$ is adopted for the interaction among carbon atoms with a slight simplification, ${ }^{18}$ and the interaction potential of $\mathrm{C}-\mathrm{O}$ and $\mathrm{O}-\mathrm{O}$ were derived from Brenner's formula. The details are described in the Appendix. A 12-6 Lennard-Jones potential is 
TABLE I. Lennard-Jones potential parameters.

\begin{tabular}{lccc}
\hline \hline$\sigma_{\mathrm{Ar}-\mathrm{C}}(\AA)$ & $\sigma_{\mathrm{Ar}-\mathrm{Ar}}(\AA)$ & $\varepsilon_{\mathrm{Ar}-\mathrm{C}}(\mathrm{J})$ & $\varepsilon_{\mathrm{Ar}-\mathrm{Ar}}(\mathrm{J})$ \\
\hline 3.385 & 3.4 & $8.013 \times 10^{-22}$ & $16.7 \times 10^{-22}$ \\
\hline \hline
\end{tabular}

applied for the carbon-argon and argon-argon interactions with the parameters in Table I.

\section{METHOD}

The impact target must be large enough in order to avoid unrealistic side effects due to the boundary condition. The diamond (111) target surface consists of a hexagonal internal full-simulated region, and a surrounding external symmetric region as shown in Fig. 1. Based on the assumption that the effects far from the impact point are not crucial to the impact phenomena, only one-sixth of the external region is simulated, and its mirror and rotated images are connected with the internal full-simulated region considering the symmetry of the crystal in order to reduce the calculation time. The internal and external regions contain 758440 and 308240 carbon atoms, respectively (2 584741 carbon atoms including virtual images without overlap). The depth parallel to the impact direction and the length of the diagonal line of the internal region are about 115 and $122 \AA$, respectively, and the external region extends by another $50 \%$ of the inner region in each direction. In the symmetric external region, the motions of carbon atoms in the symmetry center and those in the connection plane are restricted to one and two dimensions, respectively. Thereby, a discontinuity arises at the outer boundaries of the five-sixths of the internal region in contact with the virtual images, while there is no inconsis-

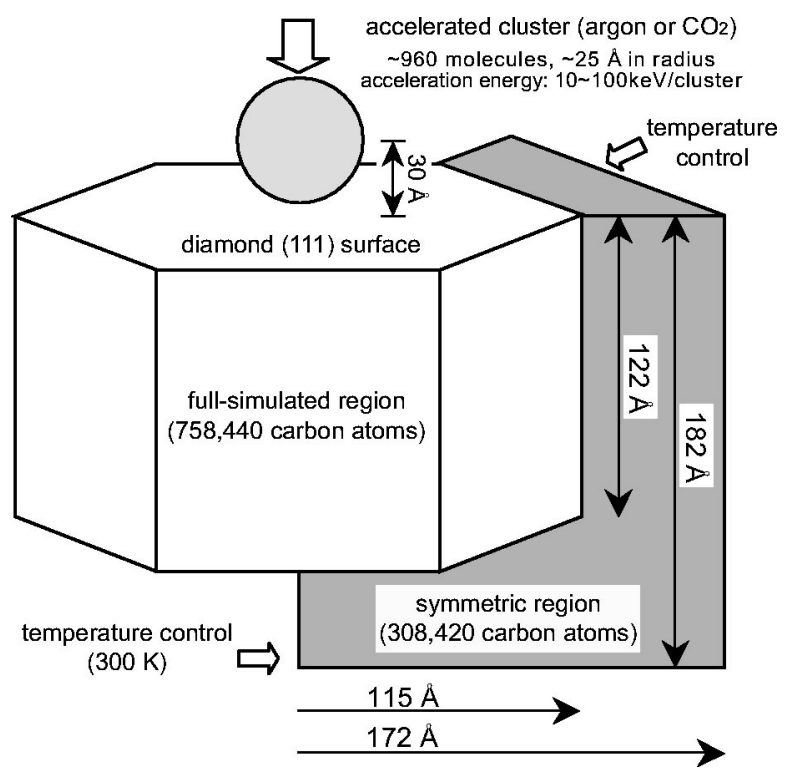

FIG. 1. System configuration of the cluster impact simulation. The diamond (111) target surface consists of a hexagonal fullsimulated internal region and a surrounding symmetric external region. Only $1 / 6$ of the external region is simulated, and its mirror and rotated images are connected to the internal region. tency in the one-sixth part of the shells in contact with the real image. In order to compensate for the problem, cylindrical shells containing the whole side interface region are considered, and the average kinetic energy of the carbon atoms in the inconsistent five-sixths part are controlled with the velocity scaling based on that of the consistent one-sixth part in each shell. An unfavorable crucial energy discontinuity can be avoided with this method. The cylindrical shells are four and 20 layered in radial and axial directions with a thickness and a height of about 5.8 and $6.15 \AA$, respectively, and the bottom interface region is also controlled in the same manner. The outer boundary of the external region is fixed and the temperature is controlled at $300 \mathrm{~K}$ near the outer boundary with the Langevin method. The whole system was preliminary relaxed at $300 \mathrm{~K}$ before the impact.

The impact clusters were obtained by cooling liquid phase argon or $\mathrm{CO}_{2}$ cluster to $40 \mathrm{~K}$, and these clusters contain 961 atoms for argon and 960 molecules for $\mathrm{CO}_{2}$ in accordance with our experimental data. ${ }^{6}$ A 12-6 Lennard-Jones potential was also applied for the inter- $\mathrm{CO}_{2}$ interactions for this preliminary simulation, but it is omitted for the impact simulation. The cluster size is about $25 \AA$ in radius, and the initial distance from the center of the cluster to the diamond surface is $30 \AA$. The cluster impact angle is perpendicular to the diamond surface, and the impact velocity depends on the acceleration energy $E_{a}$ (/cluster). Each simulation ran for 4, 8 , or $30 \mathrm{ps}$ and $4 \mathrm{ps}$, respectively. Verlet's method was adopted to integrate the equation of motion with a time step $\Delta t=0.2$ fs for the first $2 \mathrm{ps}$, and $\Delta t=0.5$ fs afterward.

\section{RESULTS AND DISCUSSIONS}

\section{A. High energy argon cluster impact}

Figure 2 shows snapshots of the argon cluster impact with the acceleration energy $E_{a}=100 \mathrm{keV}$ up to $8 \mathrm{ps}$ after the impact. A cross section parallel to the impact direction with a thickness of $10 \AA$ is shown, and carbon atoms with larger velocities have darker shades. The acceleration energy is the same as our experimental condition. ${ }^{6}$ Some color movies are also available at our web site. ${ }^{19}$ The cluster crushes into the diamond surface [Fig. 2(a)] and a typical hemispherical crater is formed about $0.7 \mathrm{ps}$ after the impact [Fig. 2(b)]. At the very beginning, the crystal structure is still reflected in the asymmetric and somewhat skew appearance of the cross sections of the crater and of the compressed (dark) region, while hemisphericity develops only at later times. Two- or threelayered asymmetric shockwaves are simultaneously induced by the impact, and they propagate in the $(\overline{11} \overline{1}),(1 \overline{1}),(\overline{1} 1 \overline{1})$, and (111) directions while keeping rather flat structures. ${ }^{19}$ They are also well realized in the external symmetric region [Fig. 2(c)]. The crater is, however, immediately filled up with the fluidized hot carbon material compressed around the impact crater due to the elastic recovery before the reflected shockwave of (111) direction seen in Fig. 2(d) as an inwardly bend structure returns from the bottom boundary. The reflected shockwave goes up through the impact point at about 3.0 ps and the surface is somehow mounded up, but it simply passes through the region and no significant effect can be seen because the impact point is still hot and is not yet 


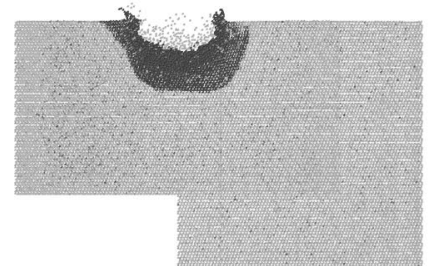

(a) $0.3 \mathrm{ps}$

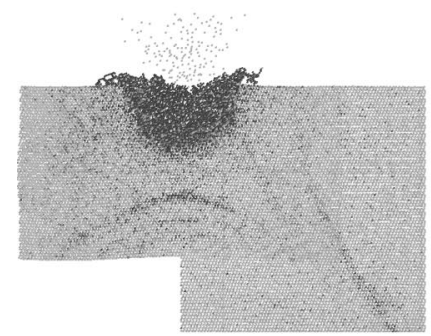

(d) $2.0 \mathrm{ps}$

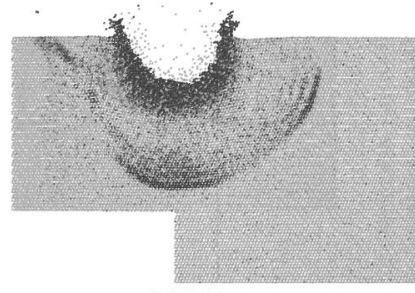

(b) $0.7 \mathrm{ps}$

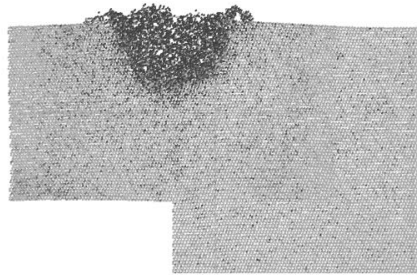

(e) $3.0 \mathrm{ps}$

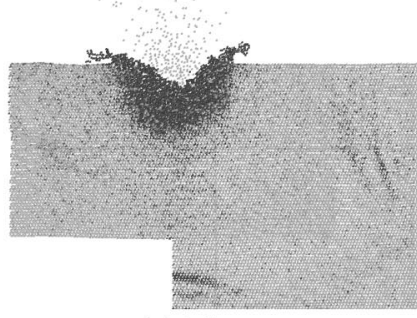

(c) $1.5 \mathrm{ps}$

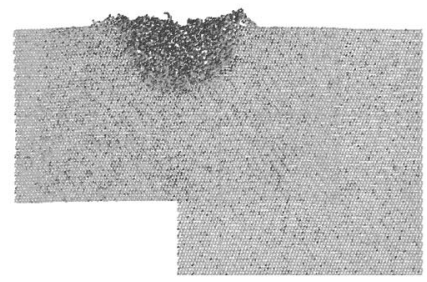

(f) $8.0 \mathrm{ps}$

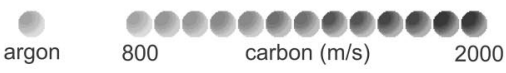

FIG. 2. Snapshots of argon cluster impact simulation up to $8 \mathrm{ps}$ after the impact with the acceleration energy $E_{a}=100 \mathrm{keV}$, where the cross sections parallel to the impact direction with a thickness of $10 \AA$ are exhibited. The brightness of carbon atoms corresponds to the velocity.

recrystallized [Fig. 2(e)]. It is technically possible to eliminate the effect of the reflected shockwave by damping the external region for a short time when the shockwave is in the external region, and we have actually carried out that simulation by damping at $1.2 \mathrm{ps}$, however, no clear difference could be seen.

The shockwave propagates with a velocity of about $14-15 \mathrm{~km} / \mathrm{s}$, a little slower than $18 \mathrm{~km} / \mathrm{s}$ in diamond, ${ }^{20}$ and this may be due to the problem with the potential parameters that give a lower force constant for the C-C bond. ${ }^{17}$ The transient crater becomes the largest at about 0.7 ps with a diameter of about $60 \AA$, and this corresponds well to the size expected from the extrapolation of macroscopic ballistic experiments, assuming a Brinell hardness number $B$ of 6000 (HB) for diamond. ${ }^{21}$

Figure 3 shows the energy profile of the system for the first $1.5 \mathrm{ps}$, where $E_{k}$ and $E_{p}$ represent the total kinetic and potential energy as the offset from the initial values, and the suffixes Ar, int, and ext are for argon atoms and for carbon atoms in the internal full-simulated and external symmetric regions, respectively. The impact energy is at first transferred mainly to the kinetic energy $E_{k}^{\text {int }}$ until about $0.3 \mathrm{ps}$, and then, the potential energy $E_{p}^{\text {int }}$ increases. $E_{p}^{\text {int }}$ reaches a maximum at about 0.7 ps when the crater size becomes almost the largest, and that time is long before the reflected shockwave comes back. Therefore, it is clear that the crater is relaxed not due to the reflected shockwave, but due to the local elastic rebound. The shockwave propagates out of the internal region at about $0.8 \mathrm{ps}$, and kinetic and potential energies of the external region are excited; then the shock reflects at the end boundary at about $1.3 \mathrm{ps}$. $E_{k}^{\mathrm{Ar}}$ approaches about $6 \mathrm{keV}$ after the impact, i.e. about $94 \%$ of the initial impact energy of $100 \mathrm{keV}$ is transferred into the target, and the impact itself is evidently classified as an inelastic process. The dependence of the remaining kinetic energy of outgoing argon atoms $* E_{k}^{\mathrm{Ar}}$ and the kinetic energy transfer to the outer region $\Delta E_{k}^{\text {ext }}$ on the impact energy $E_{a}$ is further investigated in Sec. III C.

Figure 4 shows enlarged snapshots and axisymmetric temperature distributions at 3.0 and $8.0 \mathrm{ps}$ after the impact, where the temperature distribution is calculated as an azimuthal average. The distribution is almost hemispherical. The highest temperature at the impact point rises up to about

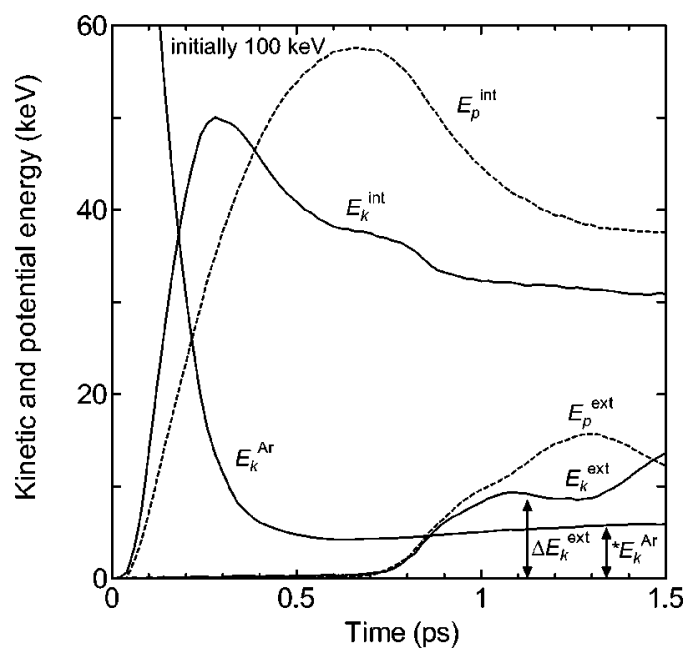

FIG. 3. Kinetic and potential energy profiles of argon cluster impact with $E_{a}=100 \mathrm{keV} . E_{k}$ and $E_{p}$ represent the total kinetic and potential energies as the offset from the initial values, and the suffixes Ar, int, and ext are for argon atoms and carbon atoms in the full-simulated and symmetric regions, respectively. 

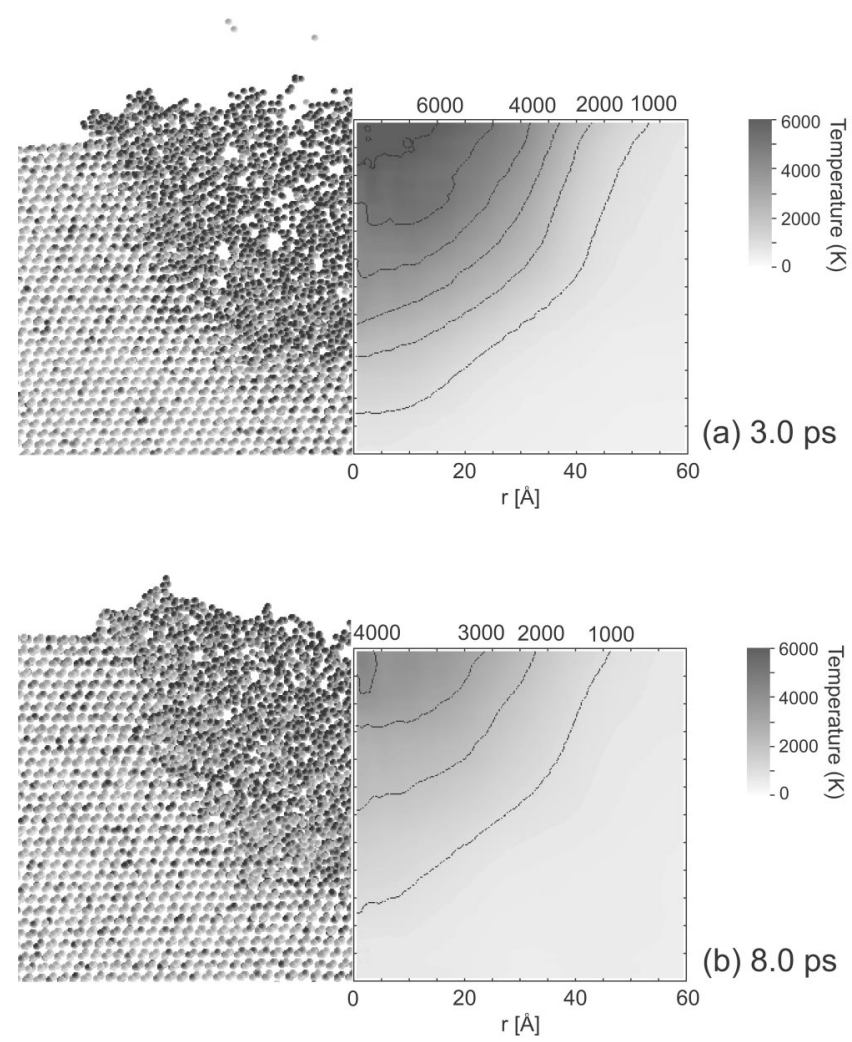

FIG. 4. Enlarged snapshots and axisymmetric temperature distributions around the impact point at (a) $3.0 \mathrm{ps}$ and (b) $8.0 \mathrm{ps}$ after the impact for argon cluster impact with $E_{a}=100 \mathrm{keV}$.

$6000 \mathrm{~K}$ at $3.0 \mathrm{ps}$ [Fig. 4(a)], but the heat rapidly dissipates into diamond due to the high thermal conductivity, and the highest temperature decreases down to under $4000 \mathrm{~K}$ at 8.0 ps, as shown in Fig. 4(b). The thermal conductivity of dia- mond using the same potential was simulated to be about $1300-1400(\mathrm{~K} / \mathrm{m} \mathrm{s})$, and that corresponds well to the experimental data. ${ }^{20}$ Thus this single impact phenomenon on diamond can be expressed as a very fast heating and cooling down process due to the extremely high thermal conductivity.

\section{B. Comparison of argon and $\mathrm{CO}_{2}$ cluster impacts}

Figure 5 shows a comparison of the enlarged snapshots of the argon and $\mathrm{CO}_{2}$ cluster impact simulations, where the shade profile for carbon atoms is the same as in Fig. 2, and oxygen atoms in the $\mathrm{CO}_{2}$ cluster impact have the same shade as argon atoms. The horizontal width is $120 \AA$, in accordance with the temperature distribution in Fig. 4. No significant difference can be observed in the structures of craters at 0.7 ps [Fig. 5(a1,b1)] because the primary impact stage is simply a hard collision process pushing the solid surface with molecules of similar masses (Ar: $\sim 40 \mathrm{amu} ; \mathrm{CO}_{2}$ : $\sim 44 \mathrm{amu}$ ). Therefore, the structures and velocities of the shockwaves are also almost the same. A clear difference appears in the collective motion of the surface after the crater formation. For the argon cluster impact, the emission of carbon atoms from the surface is strongly suppressed in the vertical direction by the argon atoms themselves as shown in Fig. 5(a2-3), and carbon atoms can escape only from the edge of the impact point. Thus, the impact surface seems rather smooth. On the other hand, the dissociated carbon and oxygen atoms stimulate the emission by taking carbon atoms away from the surface for the $\mathrm{CO}_{2}$ cluster impact as shown in Fig. 5(b2-3). After the impact species left from the surface, a remarkable difference between the two impacts can also be seen in the roughness of the surface, as in Fig. 5(a4,b4).

The numbers of carbon atoms emitted out of the diamond surface $N_{\mathrm{C}}^{\text {out }}$ for both impacts are shown in Fig. 6, where the

(a) Argon cluster impact

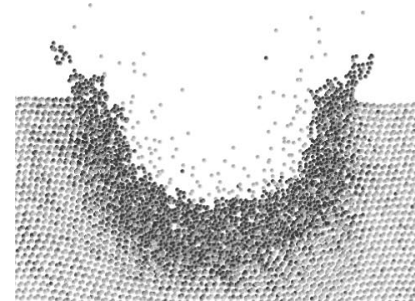

(1) $0.7 \mathrm{ps}$

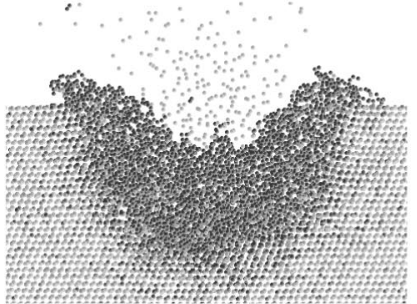

(2) $1.5 \mathrm{ps}$

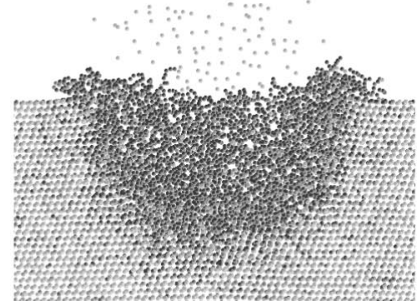

(3) $2.0 \mathrm{ps}$

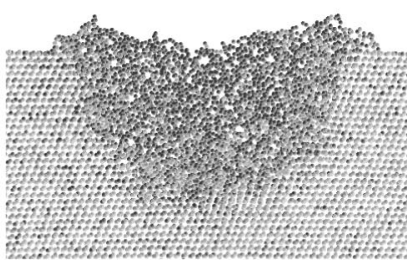

(4) $8.0 \mathrm{ps}$

(b) $\mathrm{CO}_{2}$ cluster impact

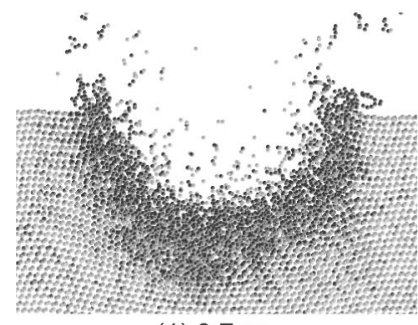

(1) $0.7 \mathrm{ps}$

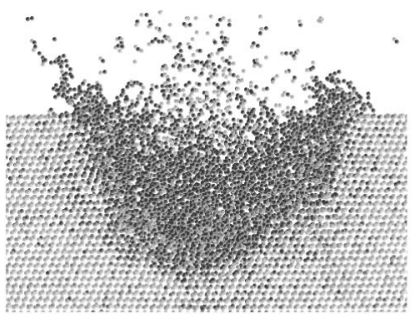

(2) $1.5 \mathrm{ps}$

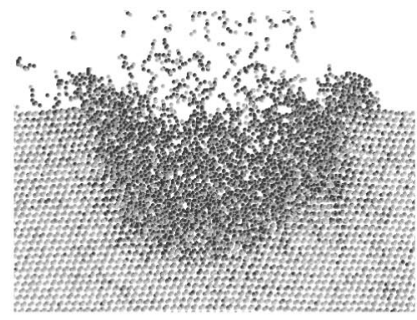

(3) $2.0 \mathrm{ps}$

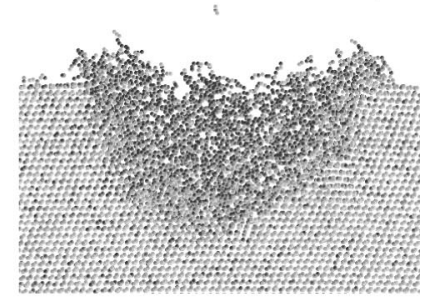

(4) $8.0 \mathrm{ps}$

FIG. 5. Comparison of (a) argon and (b) $\mathrm{CO}_{2}$ cluster impacts with $E_{a}=100 \mathrm{keV}$ at (1) $0.7 \mathrm{ps}$, (2) $1.5 \mathrm{ps}$, (3) $2.0 \mathrm{ps}$ and (4) 8.0 ps after the impact (cross section, enlarged; thickness $10 \AA$ ). 


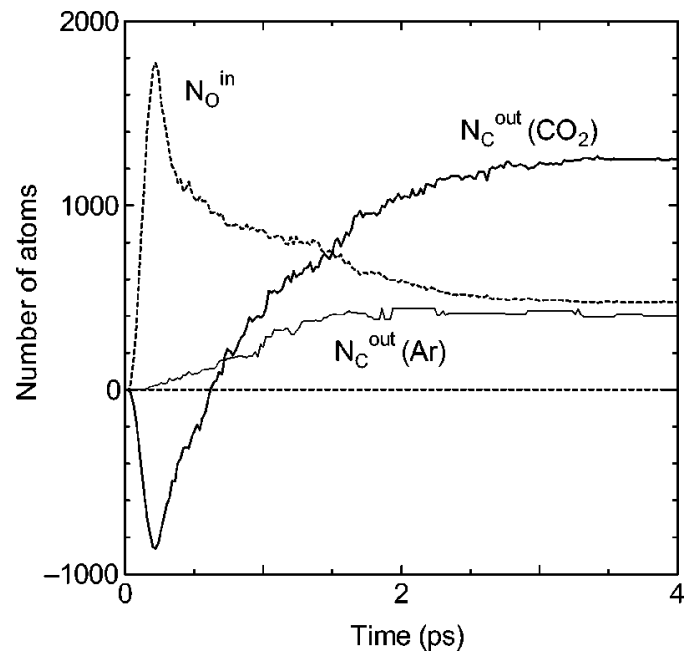

FIG. 6. Number of carbon atoms emitted out of the surface $N_{\mathrm{C}}^{\text {out }}$, and oxygen atoms remaining on the surface $N_{\mathrm{O}}^{\mathrm{in}} \cdot N_{\mathrm{C}}^{\text {out }}\left(\mathrm{CO}_{2}\right)$ is calculated as the offset from the initial number of the target atoms.

number is defined as the offset from the initial number of the target atoms. The number of oxygen atoms staying on the diamond surface $N_{\mathrm{O}}^{\text {in }}$ is also shown for the $\mathrm{CO}_{2}$ cluster impact. A bond is determined by a cutoff length for the carbonoxygen system in the potential functions used here, and an emitted cluster can be defined as a cluster not sharing any bond with the diamond surface. Although almost all of the carbon atoms in the impact $\mathrm{CO}_{2}$ cluster are first embedded into the diamond surface and $N_{\mathrm{C}}^{\text {out }}\left(\mathrm{CO}_{2}\right)$ has a negative value before about $0.7 \mathrm{ps}$, the dissociated species strongly enhances the emission afterward, and the final net number of carbon atoms emitted from the surface is more than three times larger for the $\mathrm{CO}_{2}$ cluster impact than that for the argon cluster impact. Our experiment of multiple impact resulted in the difference in the erosion effect with the factor of about $4,7,8$ and that corresponds well to the result of the single impact simulation. It is also notable that not a few oxygen atoms stay on the surface after the impact. In addition to the fact that the temperature around the impact point is lower for the $\mathrm{CO}_{2}$ cluster impact due to the energy release from the surface, these oxygen atoms may also disturb the ordered recrystallization. These two factors can both be important for the differences in the surface roughness observed in the experiment. ${ }^{7}$

Figure 7 shows the axisymmetric local potential energy distribution (upper parts of the figure), height contours (middle parts), and enlarged snapshots (lower parts) of the surface for the argon and $\mathrm{CO}_{2}$ impacts at 30 ps after the impact, where the potential energy distribution is calculated as an azimuthal average, and the local potential energy of an atom $i$ is calculated as $\sum_{j(\neq i)}\left[V_{R}\left(r_{i j}\right)+V_{A}\left(r_{i j}\right)\right] / 2$ (see the Appendix). The temperature around the impact point is under $1000 \mathrm{~K}$ at the time for both, and the recrystallization process in a short time scale has already finished. The surface for the argon cluster impact [Fig. 7(a)] is obviously smoother than that for the $\mathrm{CO}_{2}$ cluster impact [Fig. 7(b)], as expected above. In addition to the apparent difference in the roughness, a remarkable central peak structure can be seen in the
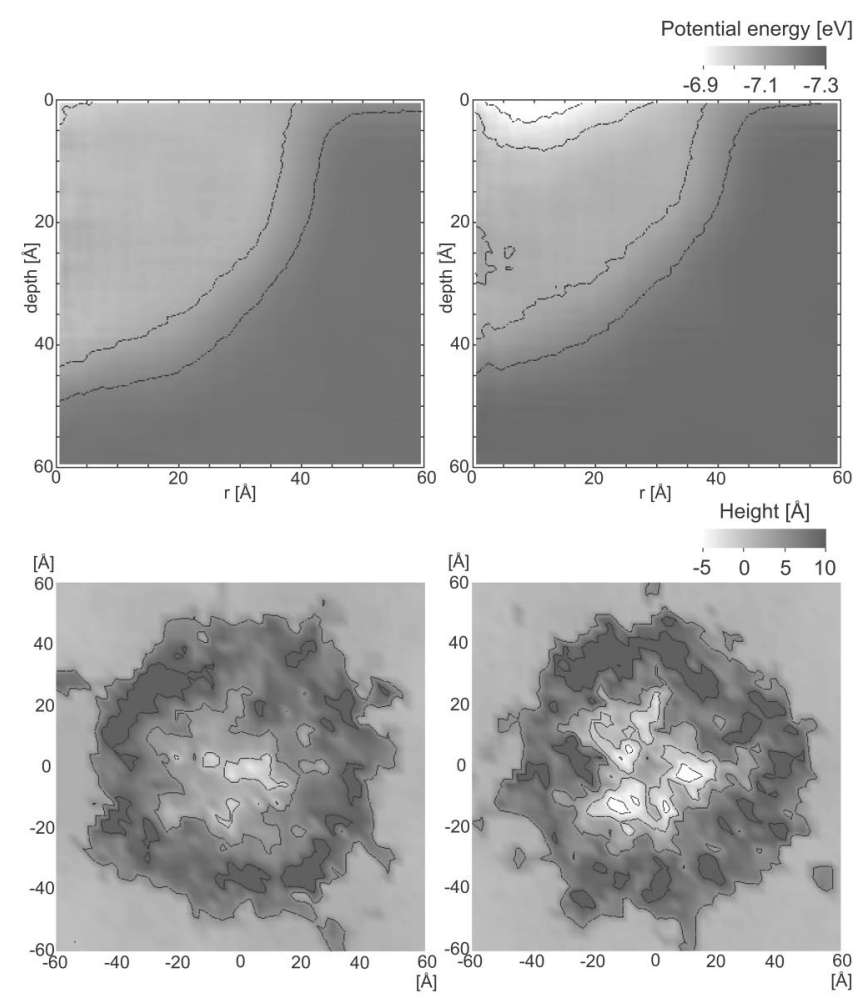

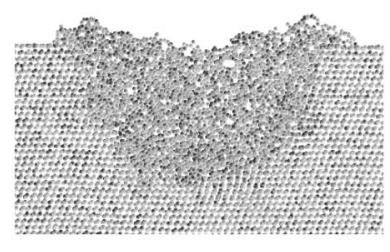

(a) Argon

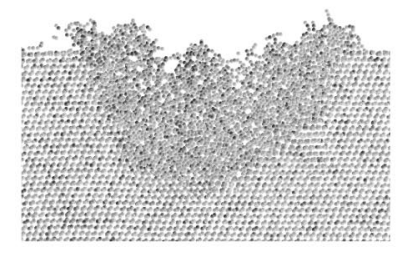

(b) $\mathrm{CO}_{2}$
FIG. 7. Axisymmetric local potential energy distribution (upper), height contours (middle) and enlarged snapshots (lower) of the surface for the argon and $\mathrm{CO}_{2}$ impacts at $30 \mathrm{ps}$ after the impact with $E_{a}=100 \mathrm{keV}$, where the potential energy distribution is calculated as an azimuthal average.

potential distribution, the height contour, and the snapshot, with a height of about $10 \AA$ only for the $\mathrm{CO}_{2}$ cluster impact.

Similar central peak structures are rather common in planetary impact craters, ${ }^{22}$ which are then called "complex" craters (e.g. crater Yuty on planet Mars, or the giant crater Herschel on Saturn's moon Mimas). In droplet collisions, or when droplets fall into a pool of liquid, transitory rebound peaks are well known, too, and show up in simulations utilizing, e.g., the volume-of-fluid Navier-Stokes method. ${ }^{23}$ With solid targets, however, hypersonic impact velocities are needed to fluidize the target material whose ensuing solidification may conserve the central peak structure. ${ }^{24}$ Then the question arises of why the central peak is formed only for $\mathrm{CO}_{2}$ impacts.

Figure 8 shows enlarged snapshots and axisymmetric velocity distributions of the carbon atoms for the argon and $\mathrm{CO}_{2}$ impacts during the crater recovery process at $1.4 \mathrm{ps}$ after the impact, where the velocity distribution is calculated as an azimuthal average of the radial and axial velocity components. A pronounced rebound flow to the vertical direction to pull up the fluidized surface can be seen in Fig. 8(b) for 


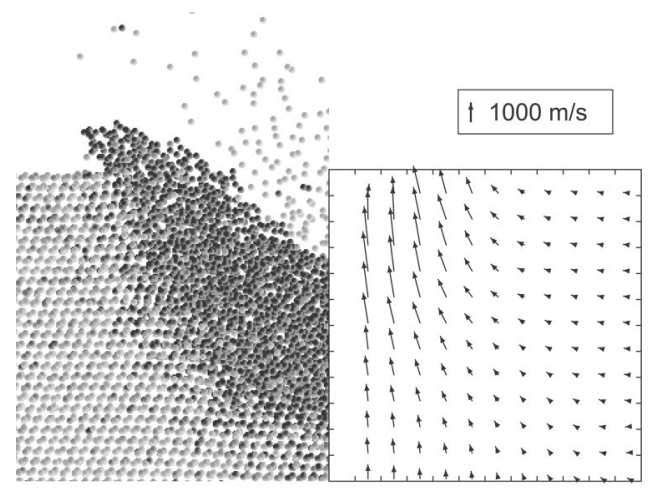

(a) Argon

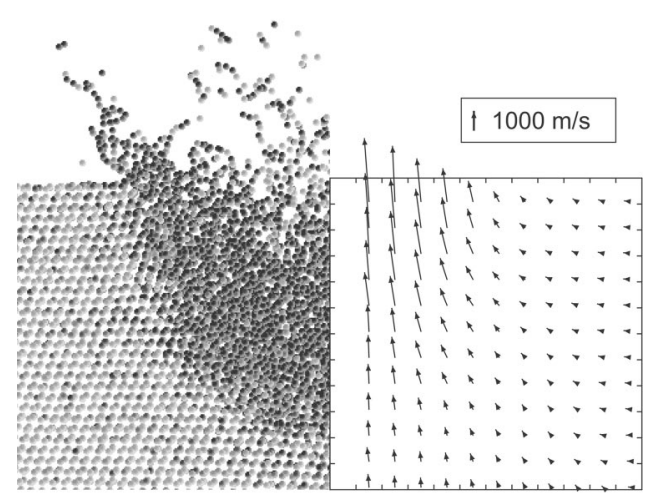

(b) $\mathrm{CO}_{2}$

FIG. 8. Snapshots and axisymmetric velocity distributions of carbon atoms for (a) argon and (b) $\mathrm{CO}_{2}$ impacts at $1.4 \mathrm{ps}$ after the impact with $E_{a}=100 \mathrm{keV}$.

the $\mathrm{CO}_{2}$ impact, and this finally results in the formation of the central peak. On the other hand, the flow seems to be suppressed by the pressure of argon as shown in Fig. 8(a) because the rebound motion of argon atoms is slower than that of carbon and oxygen atoms due to the large difference in mass between argon $(\sim 40 \mathrm{amu})$ and carbon $(\sim 12 \mathrm{amu})$ as well as oxygen $(\sim 16 \mathrm{amu})$. This pressure can also be addressed as a cause of the smoothness of the argon cluster eroded surface.

\section{Cluster impacts with lower acceleration energies}

Figure 9 shows the comparison of the snapshots of the simulations for $\mathrm{CO}_{2}$ impacts with different acceleration energies $E_{a}$ of $10,30,50$, and $75 \mathrm{keV}$ at 0.7 and $2.0 \mathrm{ps}$ after the impact. Typical transient hemispherical craters similar to that for $E_{a}=100 \mathrm{keV}$ [Fig. 2(b)] also appear in cases of $30 \leqslant E_{a}$ $\leqslant 75 \mathrm{keV}$ at about $0.7 \mathrm{ps}$ after the impact as shown in Fig. $9(1 \mathrm{~b}-\mathrm{d})$, and the two or three layered shockwaves are also clearly observed. The propagation direction and the velocity of the shockwave are almost the same for the cases of $E_{a}$ $\geqslant 30 \mathrm{keV}$, where the slight difference in the position of the shockwave is due to the difference in the exact impact time. On the other hand, only simple elastic surface deformation without phase change can be seen for $E_{a}=10 \mathrm{keV}$ [Fig. 9(1a)], and any well-structured shockwaves are also difficult to determine. All of the impact-induced craters are immediately filled up with the fluidized carbon materials [Fig. 9(2bd)], or due to the simple elastic recovery for $E_{a}=10 \mathrm{keV}$ [Fig. 9(2a)] before $2.0 \mathrm{ps,} \mathrm{without} \mathrm{any} \mathrm{effect} \mathrm{of} \mathrm{the} \mathrm{reflected}$ shockwave. Even the first layer of the surface keeps the original crystal structure for $E_{a}=10 \mathrm{keV}$.

Figure 10 shows the volumes of the crater and the plastically deformed region, where the plastically deformed region is calculated from the potential energy distributions as in Fig. 7 based on the line of $-7.2 \mathrm{eV}$. For both argon and $\mathrm{CO}_{2}$ impacts, the volume of the crater is almost proportional to the acceleration energy. According to the results of MD simulations of a single argon cluster impact on a copper (100) surface $^{15}$ in which the cluster size was changed as a

(1) $0.7 \mathrm{ps}$
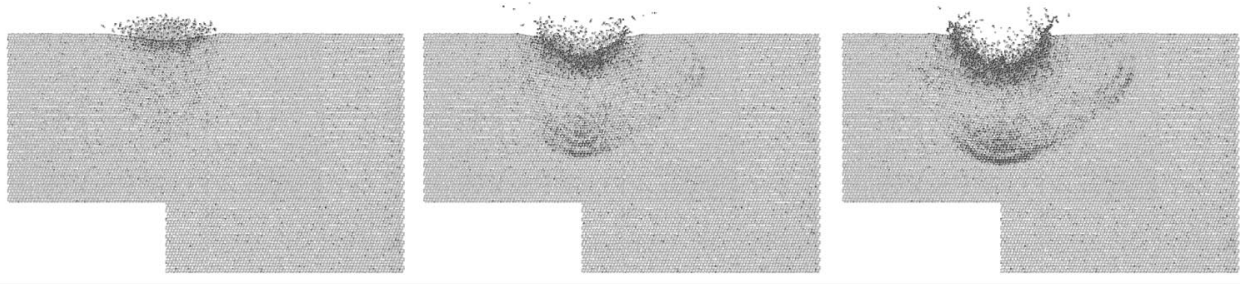

(2) $2.0 \mathrm{ps}$

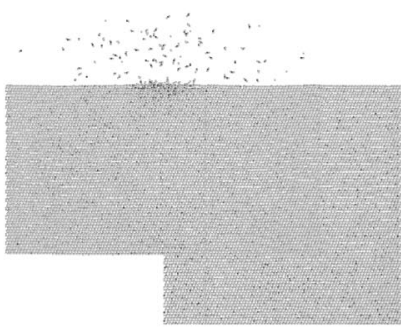

(a) $10 \mathrm{keV}$

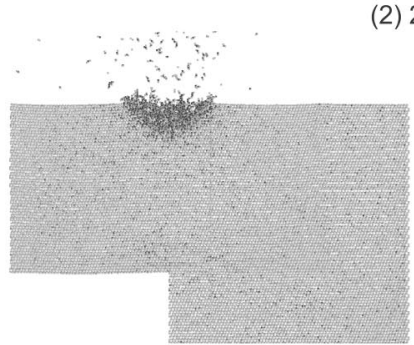

(b) $30 \mathrm{keV}$

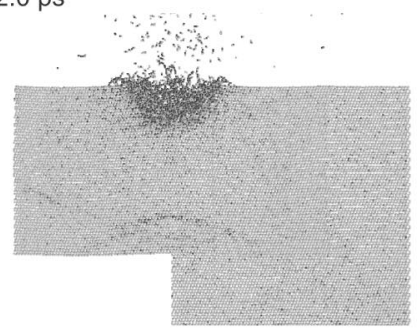

(c) $50 \mathrm{keV}$
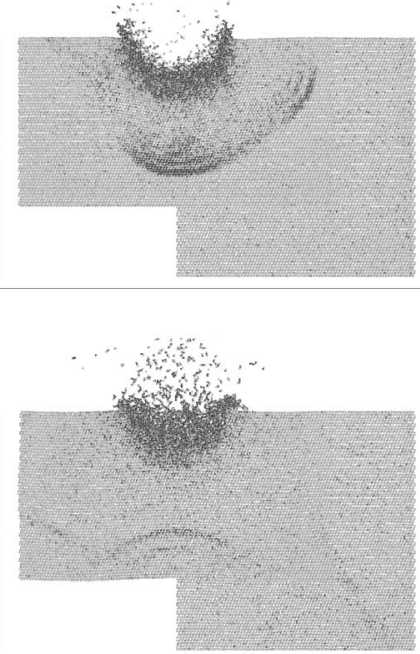

(d) $75 \mathrm{keV}$

FIG. 9. Comparison of the snapshots for $\mathrm{CO}_{2}$ impacts with different acceleration energies $E_{a}$ of (a) $10 \mathrm{keV}$, (b) $30 \mathrm{keV}$, (c) $50 \mathrm{keV}$, and (d) $75 \mathrm{keV}$ at (1) $0.7 \mathrm{ps}$ and (2) $2.0 \mathrm{ps}$ after the impact. 


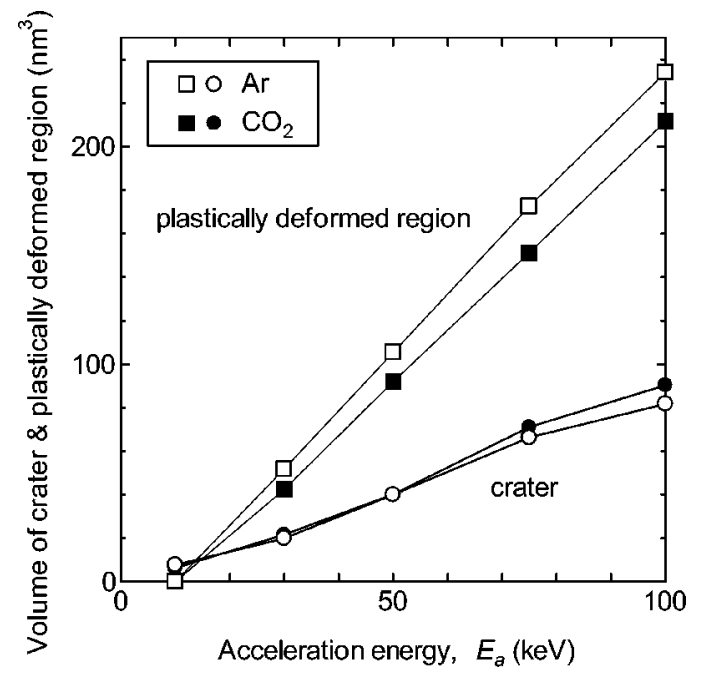

FIG. 10. Volumes of the crater and the plastically deformed region as functions of the cluster acceleration energy $E_{a}$.

parameter while keeping the acceleration energy per atom, i.e., the acceleration velocity constant, the crater depth and diameter were proportional to the one-third power of the acceleration energy. Our results show that the relation is also applicable in cases when the acceleration energy per atom is not constant, and this also corresponds well to macroscopic projectile impacts. ${ }^{21}$ In the impact simulations of $\mathrm{Ag}_{N}$ cluster on graphite, ${ }^{16}$ the implantation depth $d$ varied linearly as $E_{a} / N^{2 / 3}$. Assuming that the hole size of a layer is almost independent of the acceleration energy, and only depends linearly on the cluster cross section $\left(\propto N^{2 / 3}\right)$ in this case, the hole or crater volume $\left(\propto d N^{2 / 3}\right)$ is also proportional to the acceleration energy $E_{a}$. In our case, the crater depth was roughly proportional to the 0.65 and 0.58 powers of $E_{a}$ for argon and $\mathrm{CO}_{2}$ cluster impact, respectively, and it is still difficult to determine the relation with these results using only two different clusters. On the other hand, the volume of the plastically deformed region seems to be a linear function of the acceleration energy only for the acceleration energy above about $10 \mathrm{keV}$. In other words, a threshold acceleration energy exists in order to induce a plastic deformation on diamond.

Figure 11 shows the kinetic energy transferred to the outer-region by the shockwave $\Delta E_{k}^{\text {ext }}$ as a function of the cluster acceleration energy $E_{a}$. The remaining kinetic energy of outgoing argon atoms $* E_{k}^{\text {Ar }}$ is also shown for the argon cluster impact. $\Delta E_{k}^{\text {ext }}$ is plotted well on lines for both argon and $\mathrm{CO}_{2}$ impacts except for the data for $E_{a}=10 \mathrm{keV}$ because $\Delta E_{k}^{\mathrm{ext}}$ is too small to measure. The linear functions are expressed as $\Delta E_{k}^{\text {ext }}(\mathrm{Ar})=0.09\left\{E_{a}-11 \mathrm{keV}\right\}$ and $\Delta E_{k}^{\mathrm{ext}}\left(\mathrm{CO}_{2}\right)$ $=0.081\left\{E_{a}-13 \mathrm{keV}\right\}$. On the other hand, $* E_{k}^{\mathrm{Ar}}$ does not change much with the acceleration energy, and is almost constant about $6 \mathrm{keV}$ for $E_{a} \geqslant 30 \mathrm{keV}$. Therefore, the sum of the two kinetic energies, i.e., the acceleration energy not consumed in the inner region, is basically governed by $\Delta E_{k}^{\text {ext }}$ with a gradient of about $9 \%$. Thereby, a very high energy density condition is achieved near the impact point allowing erosion or modification especially with higher acceleration

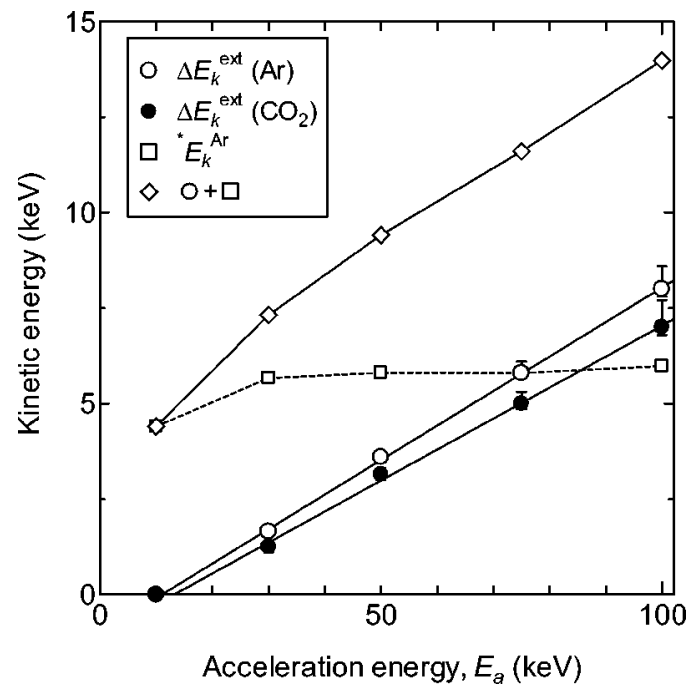

FIG. 11. Kinetic energy transferred to the outer region by the shockwave $\Delta E_{k}^{\text {ext }}$ as a function of the cluster acceleration energy $E_{a}$. The fitted lines for $\Delta E_{k}^{\text {ext }}$ s are $\Delta E_{k}^{\text {ext }}(\mathrm{Ar})=0.09\left\{E_{a}-11 \mathrm{keV}\right\}$ and $\Delta E_{k}^{\mathrm{ext}}\left(\mathrm{CO}_{2}\right)=0.081\left\{E_{a}-13 \mathrm{keV}\right\}$. $* E_{k}^{\mathrm{Ar}}$ denotes the remaining kinetic energy of outgoing argon atoms for the argon cluster impact.

energies. In addition, $\Delta E_{k}^{\text {ext }}$ is also a linear function only above around $E_{a} \geqslant 10 \mathrm{keV}$ as well as the volume of the plastically deformed region. Thus the pronounced shockwave is considered to be generated only when a plastic deformation is induced on diamond with an acceleration energy above the threshold around $10 \mathrm{keV}$. Both the volume of the plastically deformed region and $\Delta E_{k}^{\text {ext }}$ are a little larger for the argon cluster impact, and presumably, this results from the consumption of impact energy for the $\mathrm{CO}_{2}$ dissociation.

Figure 12 shows the fraction of activated $\mathrm{CO}_{2}$ molecules $F_{a}$, i.e., $\mathrm{CO}_{2}$ molecules not remaining intact, and the number of emitted carbon atoms $N_{\mathrm{C}}^{\text {out }}$ as the offset from the initial value. Only less than $10 \%$ of the $\mathrm{CO}_{2}$ molecules are activated at $E_{a}=10 \mathrm{keV}$, and no erosion effect can be seen for

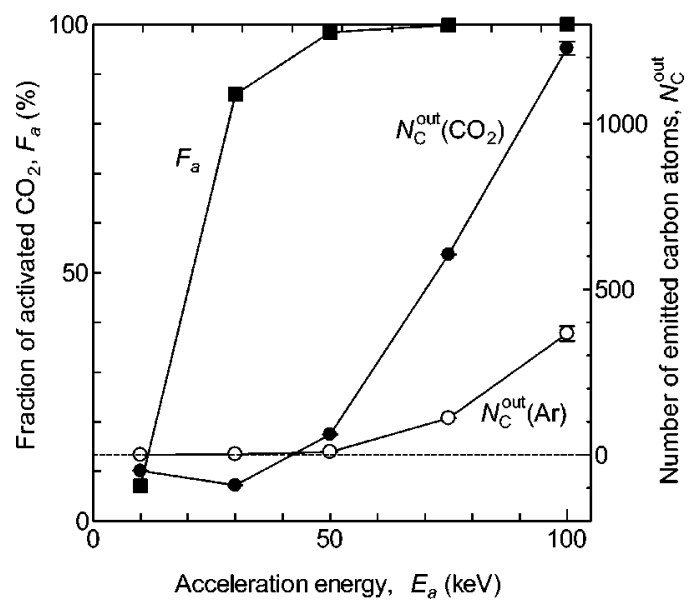

FIG. 12. Fraction of activated $\mathrm{CO}_{2}$ molecules $F_{a}$, i.e., $\mathrm{CO}_{2}$ molecules not remaining intact, and number of emitted carbon atoms $N_{\mathrm{C}}^{\text {out }}$ as the offset from the initial value. 

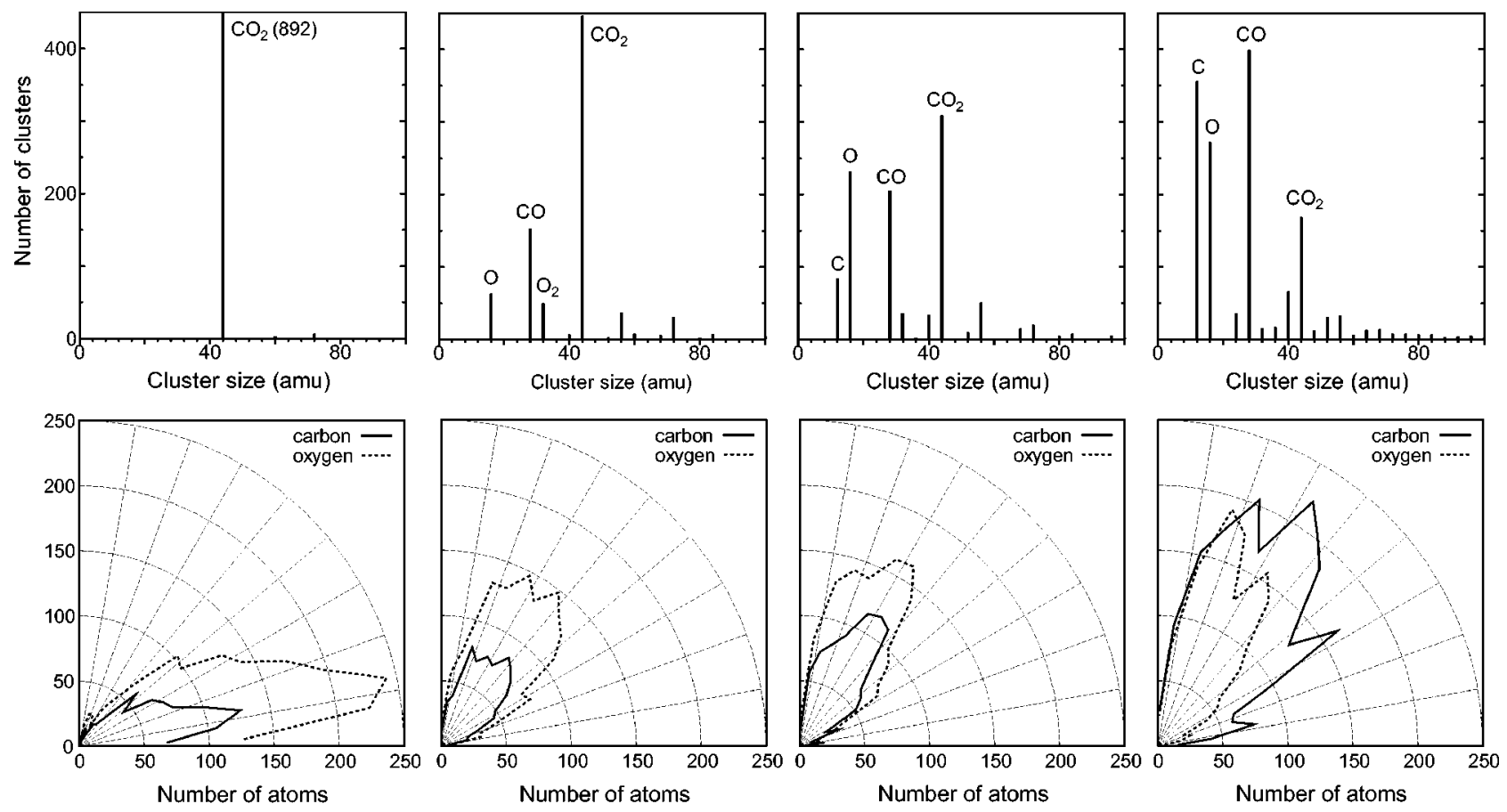

(a) $10 \mathrm{keV}$

(b) $30 \mathrm{keV}$

(c) $50 \mathrm{keV}$

(d) $100 \mathrm{keV}$

FIG. 13. Cluster size distribution (upper) and emission angle (lower) of the fragments for $\mathrm{CO}_{2}$ impacts with acceleration energies $E_{a}$ of (a) $10 \mathrm{keV}$, (b) $30 \mathrm{keV}$, (c) $50 \mathrm{keV}$, and (d) $100 \mathrm{keV}$.

both clusters. Some of the $\mathrm{CO}_{2}$ molecules simply stay on the surface without chemisorption keeping the original $\mathrm{CO}_{2}$ structure, and therefore, $N_{\mathrm{C}}^{\text {out }}\left(\mathrm{CO}_{2}\right)$ is below zero. More than $80 \%$ of the $\mathrm{CO}_{2}$ molecules are once dissociated by the impact already at $E_{a}=30 \mathrm{keV}$; however, the activated species have no excess energy to escape from the surface, and that results in deposition instead of emission. Also the argon cluster cannot stimulate the emission from the surface. Almost all $\mathrm{CO}_{2}$ molecules are activated at $E_{a}=50 \mathrm{keV}$; nevertheless, $N_{\mathrm{C}}^{\text {out }}\left(\mathrm{CO}_{2}\right)$ is still very small at this impact energy, and $N_{\mathrm{C}}^{\text {out }}(\mathrm{Ar})$ is equal to almost zero as well. The emission can only be strongly enhanced with higher acceleration energy of $E_{a} \geqslant 75 \mathrm{keV}$, and the difference in the erosion effect between argon and $\mathrm{CO}_{2}$ clusters becomes also pronounced because the dissociated $\mathrm{CO}_{2}$ species strongly activate the surface material to enhance the emission.

Figure 13 shows the cluster size distribution and the emission angle of the fragments for the $\mathrm{CO}_{2}$ impacts. At $E_{a}$ $=10 \mathrm{keV}$, almost only $\mathrm{CO}_{2}$ molecules can be seen [Fig. 13(a)], which simply impinge onto the surface and reflect back without reaction. In this case, the reflection angle is nearly glancing to the surface. $\mathrm{CO}_{2}$ molecules are still dominant with a lower acceleration energy of $E_{a}=30 \mathrm{keV}$ [Fig. 13(b)], though more than $80 \%$ of the original $\mathrm{CO}_{2}$ species do not keep the original composition as described in Fig. 12. The impact $\mathrm{CO}_{2}$ species once dissociate into $\mathrm{CO}$ and $\mathrm{O}$ at first, and then recombine into $\mathrm{CO}_{2}$ again without a strong effect on emission. Thus, some cluster signals such as $\mathrm{O}$, $\mathrm{CO}$, and $\mathrm{O}_{2}$ originating from these two components are also observed, while almost no signal can be seen at the carbon atom mass. The emission angle is around $30^{\circ}$, and is very different from the case of $E_{a}=10 \mathrm{keV}$. Various signals appear at $E_{a}=50 \mathrm{keV}$ [Fig. 13(c)], including carbon atoms, however, the impact cannot induce more emission of the surface carbon material, and the signals at $\mathrm{O}, \mathrm{CO}$, and $\mathrm{CO}_{2}$ are still intense. The emission angle is also similar to the case of $E_{a}=30 \mathrm{keV}$. At $E_{a}=100 \mathrm{keV}$ [Fig. 13(c)], more intense signals of $\mathrm{C}$ and $\mathrm{CO}$ appear, and that is clearly due to the strong emission of the surface carbon material. It is also notable that the signal of $\mathrm{O}_{2}$ (right neighbor of $\mathrm{CO}$ ) becomes smaller with the increase of the acceleration energy, because the oxygen atoms are eager to react with carbon species. The emission angle is also around $30^{\circ}$ although it is more widely distributed compared to the cases of $E_{a}=30$ and $50 \mathrm{keV}$, and the signals more glancing to the surface seem to be due to the direct fragment edged out without reaction at a very early stage of the impact. The active emission fragments may lead to re-deposition on vertical surfaces when applying this erosion technique to micro- or nanofabrication.

\section{CONCLUDING REMARKS}

Large-scale molecular dynamics simulations of single cluster impacts on a diamond surface were performed in order to investigate the surface erosion process. For the acceleration energy $E_{a}$ of $100 \mathrm{keV}$, a hemispherical crater and shockwaves are created after the impact, but the crater is immediately replenished with the fluidized hot carbon material. Compared to the argon cluster impact, the $\mathrm{CO}_{2}$ cluster impact induces significant emission from the surface at $E_{a}$ $=100 \mathrm{keV}$. As a long time effect, a central peak is formed only for the $\mathrm{CO}_{2}$ cluster impact, and that is ascribed to the 
TABLE II. Potential parameters for oxygen related interactions. All parameters not given are the same as in the original C-C potential function in Table I of Ref. 17.

\begin{tabular}{lcccc}
\hline \hline & $D_{e}(\mathrm{eV})$ & $S$ & $\beta(1 / \AA)$ & $R_{e}(\AA)$ \\
\hline $\mathrm{C}-\mathrm{O}$ & 12.04 & 1.11 & 2.3 & 1.1 \\
$\mathrm{O}-\mathrm{O}$ & 14.92 & 1.056 & 3.043 & 0.9665 \\
& & & & \\
$a_{1}$ & $b_{1}$ & $c_{1}$ & $d_{1}$ & $g_{0}$ \\
\hline $7.77 \times 10^{-2}$ & $1.342 \times 10^{-1}$ & 1.673 & $1.17 \times 10^{-2}$ & 1.073 \\
\hline \hline
\end{tabular}

strong backward flow pulling up the surface. Transient craters are also observed for a lower acceleration energy of 30 $\leqslant E_{a} \leqslant 75 \mathrm{keV}$, while only simple elastic deformation is seen for $E_{a}=10 \mathrm{keV}$. The volume of the transient crater is almost proportional to $E_{a}$ while the volume of the plastically deformed region and the kinetic energy transfer via the shockwave are linear functions of $E_{a}$ only above a threshold of about $10 \mathrm{keV}$. An appreciable surface erosion is observed only for $E_{a} \geqslant 75 \mathrm{keV}$, where the chemical enhancement causes a strong peak of $\mathrm{CO}$ in the size distribution of the emitted fragments for the $\mathrm{CO}_{2}$ cluster impacts, thus proving the reactive accelerated cluster erosion concept.

\section{ACKNOWLEDGMENTS}

This work was supported by the Forschungszentrum Karlsruhe, Technik und Umwelt, Germany, and by a Grantin-Aid for JSPS Fellows (No. 11-08826) from the Ministry of Education, Science, Sports and Culture, Japan.

\section{APPENDIX POTENTIAL FUNCTION}

The empirical potential function proposed by Brenner ${ }^{17}$ using the parameters in potential $\mathrm{I}$ is applied. The conjugatecompensation term $F$ in the original function is ignored here: ${ }^{18}$

$$
\begin{gathered}
E_{b}=\sum_{i} \sum_{j(>i)}\left[V_{R}\left(r_{i j}\right)+V_{A}\left(r_{i j}\right)\right] \\
V_{R}\left(r_{i j}\right)=f\left(r_{i j}\right) \frac{D_{e}}{S-1} \exp \left\{-\beta \sqrt{2 S}\left(r_{i j}-R_{e}\right)\right\} \\
V_{A}\left(r_{i j}\right)=-B^{*} f\left(r_{i j}\right) \frac{D_{e} S}{S-1} \exp \left\{-\beta \sqrt{2 / S}\left(r_{i j}-R_{e}\right)\right\}, \\
B^{*}=\frac{B_{i j}+B_{j i}}{2}, B_{i j}=\left[1+\sum_{k(\neq i, j)} G_{c}\left(\theta_{i j k}\right) f\left(r_{i k}\right)\right]^{-\delta} \\
G_{c}(\theta)=a_{0}\left(1+\frac{c_{0}^{2}}{d_{0}^{2}}-\frac{c_{0}^{2}}{d_{0}^{2}+(1+\cos \theta)^{2}}\right)
\end{gathered}
$$

TABLE III. Carbon-oxygen and oxygen-oxygen binding energy $D_{e q}$ (in eV) and bond length $R_{e q}$ for some compounds given by the potential and experimental data (Ref. 25).

\begin{tabular}{lcccc}
\hline \multirow{2}{*}{ Compound } & \multicolumn{2}{c}{$D_{e q}(\mathrm{eV})$} & \multicolumn{2}{c}{$R_{e q}(\AA)$} \\
$\mathrm{CO}$ & Calc. & Expt. & Calc. & Expt. \\
$\mathrm{CO}_{2}$ & 9.10 & 11.16 & 1.18 & 1.13 \\
$\mathrm{C}-\mathrm{O}$ (single bond) & 8.67 & 8.34 & 1.20 & 1.16 \\
\hline $\mathrm{O}_{2}$ & 3.51 & 3.71 & 1.46 & 1.43 \\
$\mathrm{O}-\mathrm{O}$ (single bond) & 5.14 & 5.16 & 1.21 & 1.21 \\
\hline \hline
\end{tabular}

where $E_{b}, V_{R}, V_{A} r_{i j}$, and $f(r)$ denote the total potential energy of the system, Morse-type repulsive and attractive terms, the distance between carbon atoms $i$ and $j$, and the cutoff function, respectively.

The interaction potential of $\mathrm{C}-\mathrm{O}$ and $\mathrm{O}-\mathrm{O}$ were derived from Brenner's formula in the following manner. Brenner's potential can express the covalent bond status of $s p, s p^{2}$, and $s p^{3}$ in a hydrocarbon network system via the bond order $B_{i j}$ in Eq. (A4) including the effect of the number of other bonds on a carbon atom and the bond angle in $G_{c}(\theta)$, e.g., a carbon atom surrounded by four hydrogen atoms is configured to a regular tetragon as the most stable location by assigning the bond angle to minimize the total potential energy. We assume that an oxygen atom has a bond structure similar to a carbon atom, i.e., the oxygen related covalent bond status is expressed via the bond order as a function of the bond angle. A basic difference between carbon and oxygen atoms is the two nonshared electron pairs of oxygen atoms, and they are treated here as two preexisting virtual bonds $\left[k \mathrm{~s}\right.$ in Eq. (A4); $\left.f\left(r_{i k}\right)=1\right]$, which are automatically located at the energetically most stable position for the real bonds. For instance, if one atom is inside the cutoff range of an oxygen atom, the interaction is considered as a real bond, then the three-bond network system of one real bond and two virtual bonds is configured in a common plane with the bond angle of $120^{\circ}$. Then, Eq. (A4) is changed as in Eq. (A6),

$$
B_{i j(i \text { :oxygen })}=\left[g_{0}+\sum_{k(\neq i, j)} G_{o}\left(\theta_{i j k}\right) f\left(r_{i k}\right)\right]^{-\delta}
$$

where $g_{0}=1+2 G_{c}\left(120^{\circ}\right) \neq 1$ even on one real-bond condition. $\delta$ and $f(r)$ are the same as in the original potential based on the assumption that the oxygen bonding system is the same as the carbon system. Under the existence of two atoms interacting with an oxgen atom, i.e. two real bonds, the four-bond $s p^{3}$ network is formed which prefers a regular tetragon as the most stable configuration. In this case, the most stable positions of the two virtual bonds change depending on the bond angle between two real bonds. Therefore, Eq. (A7) was fitted using the cosine function of the bond angle.

$$
G_{o}(\theta)=a_{1} \cos \theta+\frac{b_{1}}{c_{1}+\cos \theta}+d_{1}
$$


Then the Morse parameters $D_{e}, S$, and $\beta$ were defined from the normal $\mathrm{C}-\mathrm{O}$ and $\mathrm{O}-\mathrm{O}$ binding energy data. The potential parameters are shown in Table II. The binding energy and bond length of some compounds are shown in Table III. One problem to be mentioned is that this potential can basically handle up to double bonds, and therefore, some special bonds like a $\mathrm{CO}$ molecule with an $\mathrm{O}^{*} \equiv \mathrm{C}^{*}$ structure are rather difficult to express.
* Now at Department of Mechanophysics Engineering, Osaka University, 2-1 Yamadaoka, Suita, Osaka 565-0871, Japan; Electronic address: yamaguchi@mech.eng.osaka-u.ac.jp; URL:http://wwwgcom.mech.eng.osaka-u.ac.jp/ yamaguchi/

${ }^{\dagger}$ Also at Forschungszentrum Karlsruhe; Electronic address: juergen.gspann@imt.fzk.de

${ }^{1}$ J. Gspann, Sens. Actuators A A51, 37 (1995).

${ }^{2}$ D. B. Fenner, J. Hautala, L. P. Allen, J. A. Greer, W. J. Skinner, and J. I. Budnick, in Fundamental Mechanisms of Low-EnergyBeam-Modified Surface Growth and Processing, edited by S. C. Moss, E. H. Chason, B. H. Cooper, J. M. E. Harper, T. Diaz de la Rubia, and M. V. Ramana Murty, MRS Symposia Proceedings No. 585 (Materials Research Society, Pittsburgh, 2000), p. 27.

${ }^{3}$ J. Gspann, Trans. Mat. Res. Soc. Japan 17, 107 (1994).

${ }^{4}$ E. W. Becker, K. Bier, and W. Henkes, Z. Phys. 146, 333 (1956).

${ }^{5}$ E. W. Becker, Laser Part. Beams 7, 743 (1989).

${ }^{6}$ A. Gruber, J. Gspann, and H. Hoffmann, Appl. Phys. A: Mater. Sci. Process. 68, 197 (1999).

${ }^{7}$ C. Becker, J. Gspann, and R. Krämer, Eur. Phys. J. D 16, 301 (2001).

${ }^{8}$ G. Becker, J. Gspann, R. Krämer, and Y. Yamaguchi, Chin. Phys. 10, 174 (2001).

${ }^{9}$ C. L. Cleveland and U. Landman, Science 257, 355 (1992).

${ }^{10}$ H. Haberland, Z. Insepov, and M. Moseler, Nucl. Instrum. Methods Phys. Res. B 153, 199 (2000).

${ }^{11}$ M. Moseler, O. Rattunde, J. Nordiek, and H. Haberland, Nucl. Instrum. Methods Phys. Res. B 164-165, 522 (2000).

${ }^{12}$ M. Henkel and H. M. Urbassek, Nucl. Instrum. Methods Phys. Res. B 145, 503 (1998).
${ }^{13}$ T. Aoki, J. Matsuo, Z. Insepov, and I. Yamada, Nucl. Instrum. Methods Phys. Res. B 121, 49 (1997).

${ }^{14}$ T. J. Colla, R. Aderjan, R. Kissel, and H. M. Urbassek, Phys. Rev. B 62, 8487 (2000).

${ }^{15}$ Z. Insepov, R. Manory, J. Matsuo, and I. Yamada, Phys. Rev. B 61, 8744 (2000).

${ }^{16}$ S. J. Carroll, P. D. Nellist, R. E. Palmer, S. Hobday, and R. Smith, Phys. Rev. Lett. 84, 2654 (2000).

${ }^{17}$ D. W. Brenner, Phys. Rev. B 42, 9458 (1992).

${ }^{18}$ Y. Yamaguchi and S. Maruyama, Chem. Phys. Lett. 286, 336 (1998).

${ }^{19}$ Y. Yamaguchi, http://www-gcom.mech.eng.osaka-u.ac.jp / yamaguchi /index.html.

${ }^{20}$ For example, G. Elert, http://hypertextbook.com /physics /matter /polymorphs /index.shtml (1998).

${ }^{21}$ J. Gspann, in Physics and Chemistry of Finite Systems: From Clusters to Crystals, edited by P. Jena (Kluwer, Amsterdam, 1992), p. 463.

${ }^{22}$ H. J. Melosh, Impact Cratering (Oxford University Press, Oxford, 1989).

${ }^{23}$ A. Frohn and N. Roth, Dynamics of Droplets (Springer-Verlag, Heidelberg, 2000).

${ }^{24}$ J. Gspann, M. Hase, and Y. Yamaguchi, ForschungszentrumNachrichten (Forschungszentrum Karlsruhe) 33(4), 316 (2001) (in German), accessible at FZ-Karlsruhe official site: http:// presse.fzk.de /aktuelles /nachrichten /doku04_2001.htm.

${ }^{25} \mathrm{~S}$. Han and C. Yoder, http://wulfenite.fandm.edu/Data/ Table_6.html. 